\section{DEL1: taking the bite out of periodontitis}

\section{By Michael J. Haas, Senior Writer}

An international team of researchers has used the endothelial protein DEL1 to treat periodontitis in mice. ${ }^{1}$ Despite the therapeutic angle, the near-term utility of DEL1 is more likely to be as a diagnostic and risk marker for the disease.

Periodontitis results from a chronic inflammatory response to bacterial plaque and tartar deposits on the teeth. The consequent neutrophil infiltration of the gingiva (gums) damages gingival tissues and toothsupporting alveolar bone, which leads to tooth loss.

Treatment of the condition involves lengthy and expensive procedures to remove the deposits and may be accompanied by local use of antibiotics to kill bacteria and/or systemic treatment with low doses of the generic antibiotic doxycycline. Low-dose doxycycline does not have antimicrobial properties and instead reduces bone damage by inhibiting the tissuedamaging matrix metalloproteinases (MMPs) secreted by neutrophils in the gingiva.

Figure 1. DELving into periodontitis. According to a study in Nature Immunology, therapy with EGF-like repeats and discoidin I-like domains 3 (EDIL3; DEL1) could treat periodontitis by blocking excessive inflammatory responses that cause tissue damage and tooth loss.

In the healthy gingiva, [a] bacteria induce local $\mathrm{CD} 4^{+} \mathrm{T}$ cells to produce [b] IL-17, which recruits [c] circulating neutrophils to the site of inflammation. At the endothelia of blood vessels, LFA-1 (CD11a-CD18) (blue rectangles) expressed on neutrophils binds either $[d(1)] D E L 1$, thereby preventing infiltration by neutrophils, or [d(2)] intercellular adhesion molecule-1 (ICAM-1; CD54), thereby allowing infiltration by neutrophils that $[e(1)]$ secrete matrix metalloproteinases (MMPs) and other enzymes to destroy bacteria and produce additional [b] IL-17, which, in turn, both [d(1)] downregulates DEL1 and [c] recruits more neutrophils.

Deficiency in [d(1)] DEL1 due to downregulation by [b] IL-17 or age can result in $[\mathrm{e}(2)]$ excessive neutrophil infiltration, which sets up a vicious cycle of chronic inflammation, neutrophil recruitment and increased levels of MMPs and other extracellular matrixdestroying enzymes that [f] damage gingival tissue and toothsupporting alveolar bone, leading to tooth loss.

Leukothera, an Aggregatibacter actinomycetemcomitansderived leukotoxin that binds to LFA-1 from Actinobac Biomed Inc., is in preclinical testing to treat psoriasis and leukemia.
However, up to $5 \%$ of periodontitis cases are refractory to conventional therapy, leading to recurrent disease for reasons that are not well understood. Moreover, current therapies do not address the underlying immune system response that drives the disease.

Groups at the NIH's National Cancer Institute (NCI) had previously shown that endothelial cells secrete DEL1 (EGF-like repeats and discoidin I-like domains 3; EDIL3) and elucidated a role for the protein in the intercellular adhesion processes by which circulating leukocytes infiltrate tissues. $^{2,3}$ These studies led the international team of researchers on the current study to hypothesize that DEL1 might regulate neutrophil infiltration of the gingiva in periodontitis.

The group first studied the gingiva of patients with periodontitis and those of old, normal mice and saw lower levels of DEL1 and higher levels of the leukocyte-recruiting cytokine IL-17 than those in healthy controls and young mice, respectively. Young Del1 knockout mice also had higher Il-17 levels and more neutrophil infiltration in the gingiva, and greater periodontal bone loss than age-matched, wild-type controls.

In young and old mouse models of periodontitis, local or systemic injection of Del1 or a Del1-Fc fusion protein decreased inflammation, Il-17 levels, neutrophil infiltration in the gingiva and periodontal bone loss compared with injection of bovine serum albumin or $\mathrm{FC}$ alone, respectively.

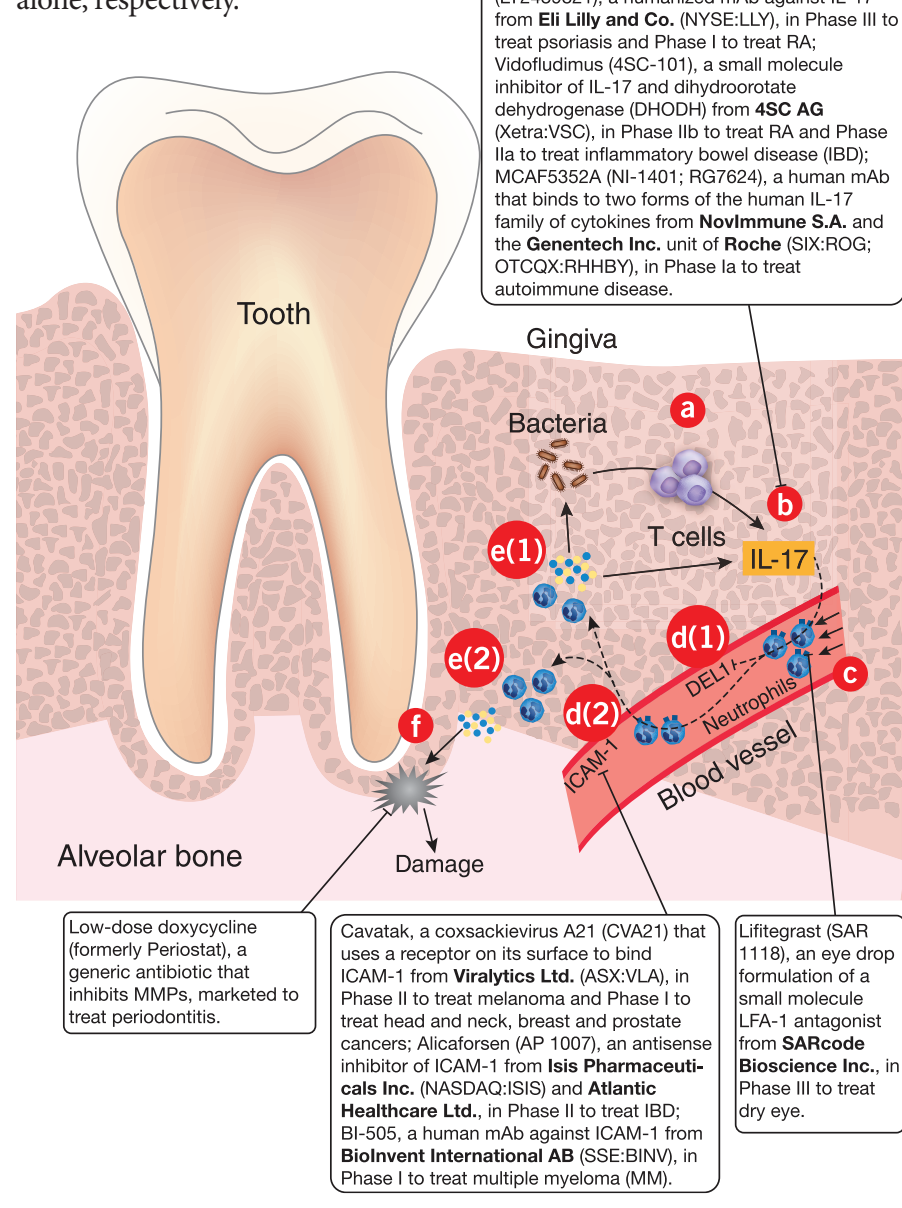

Secukinumab (AIN457), a human mAb against IL-17 from Novartis AG (NYSE:NVS; SIX:NOVN), in Phase III to treat ankylosing spondylitis, arthritis, psoriasis and rheumatoid arthritis (RA) and Phase II to treat Crohn's disease and multiple sclerosis (MS); Ixekizumab (LY2439821), a humanized mAb against IL-17 from Eli Lilly and Co. (NYSE:LLY), in Phase III to ibitimus (4SC-101), a small molecu Xetra:VSC), in Phase llb to treat RA and Phase (N) that binds to two forms of the human IL-17 amily of cytokines from Novlmmune S.A. and Genentech Inc. unit of Roche (SIX:ROG 
Additional mouse studies revealed that in healthy gingiva, DEL1 blocks interactions between intercellular adhesion molecule-1 (Icam-1; Cd54) on endothelial cells and Lfa-1 (Cd11a-Cd18) on circulating neutrophils. The result is prevention of neutrophil infiltration (see Figure 1, "DELving into periodontitis").

However, IL-17 produced by local CD4 ${ }^{+} \mathrm{T}$ cells can downregulate DEL1 and enable neutrophil infiltration of the gingiva, thus setting up a cycle of chronic inflammation and neutrophil recruitment that eventually leads to bone damage and tooth loss.

Lastly, the team showed that local administration of antagonists of IL-17, LFA-1 and ICAM-1 protected the periodontitis models from bone loss but did so less effectively than a local or systemic Del1-Fc fusion protein.

"A DEL1-derived peptide would be an ideal therapeutic" because DEL1 is an endogenous protein and thus should be safer than antibodies or small molecules that agonize the target, said team coleader George Hajishengallis. "Future studies will have to show whether such a peptide would be as effective as the full-length protein."

Hajishengallis is professor of microbiology at the University of Pennsylvania's School of Dental Medicine. When he co-led the team, he was professor of periodontics, endodontics and dental hygiene and associate director of the Center for Oral Health and Systemic Disease at the University of Louisville's School of Dentistry and professor of microbiology and immunology at the University of Louisville School of Medicine.

The team was co-led by Triantafyllos Chavakis, who headed the earlier NCI research groups and now is chief of the Division of Vascular Inflammation, Diabetes and Kidney in the Department of Internal Medicine at the Dresden University of Technology. Other researchers on the team were from the University of Ulsan, and Barts and the London School of Medicine and Dentistry, which is part of Queen Mary, University of London.

Data were reported in Nature Immunology. ${ }^{1}$

\section{DELivering diagnostics}

Because there are no methods for early diagnosis of periodontitis or for predicting a patient's response to therapy, DEL1's near-term utility is likely as a disease marker.

"This is an extremely impressive paper, and the team did an excellent job of developing and testing their hypothesis" to show that DEL1-based therapies could treat periodontitis, said Michael Glogauer, associate professor of dentistry at the University of Toronto. "Most of my own research focuses on factors released by neutrophils in the gingiva, but DEL1 prevents those cells from getting into the gingiva in the first place."

"Right now we can only diagnose periodontitis when bone damage has already occurred," he added. "DEL1 could help diagnose the disease earlier and possibly help identify individuals at risk of developing it."

He added that in patients who already have periodontitis, DEL1 might have prognostic value in helping distinguish between chronic and refractory forms of the disease.

"Chronic periodontitis responds well to the procedures for removing plaque buildup and good oral hygiene between visits to the dentist, but refractory periodontitis is very difficult to treat," he said. Glogauer noted that patients with refractory disease appear to be more sensitive to oral bacteria and thus more susceptible to the disease, which in turn increases the chance of recurrence.
"DEL1 might predict how treatable a patient's disease is and how likely it is to recur. This would be important for determining the frequency of follow-up care to manage the disease after plaque buildup has been removed," he said.

As a first step toward developing DEL1 as a disease marker, it would be important to determine the levels of DEL1 in healthy people and how these levels change as people age, Glogauer told SciBX.

Hajishengallis agreed that future studies might confirm that low DEL1 levels-and accompanying high IL-17 levels-in the gingiva could be markers for periodontitis. "But regardless of DEL1's potential use as a diagnostic tool, our mouse studies suggest that it could prevent or reverse periodontal inflammation and protect patients from bone loss."

He said a group led by Perumal Thiagarajan has developed-but not yet tested-a quantitative ELISA for measuring DEL1 levels in humans. For periodontal applications, measuring DEL1 levels in fluid secreted by the gingival epithelium, rather than saliva, would likely be the right approach, Hajishengallis said.

Thiagarajan is professor of pathology and immunology at Baylor College of Medicine.

\section{Other bones to pick}

Glogauer also wanted to know whether DEL1 is involved in autoimmune diseases that have an inflammatory component. "Osteoarthritis would be especially interesting because neutrophils play a role there and some of the disease processes are similar to those in periodontitis," he said.

"We have thought about looking at rheumatoid arthritis, and osteoarthritis should be worth looking into, though we don't know whether DEL1 is expressed in the relevant tissues," said Hajishengallis. "Regardless, local application of DEL1 might protect against inflammation even in tissues that do not ordinarily express the protein."

The team also plans to develop therapies derived from human DEL1 to prevent and treat periodontitis, he said.

The University of Louisville Research Foundation and the NIH have applied for a patent covering the findings reported in the paper. The IP is available for licensing or partnering, Hajishengallis said.

Haas, M.J. SciBX 5(15); doi:10.1038/scibx.2012.381

Published online April 12, 2012

\section{REFERENCES}

1. Eskan, M.A. et al. Nat. Immunol.; published online March 25, 2012; doi:10.1038/ni.2260

Contact: George Hajishengallis, University of Pennsylvania, Philadelphia, $\mathrm{Pa}$.

e-mail: geoh@upenn.edu

2. Choi, E.Y. et al. Science 322, 1101-1104 (2008)

3. Chavakis, E. et al. Thromb. Haemost. 102, 191-197 (2009)

COMPANIES AND INSTITUTIONS MENTIONED

Baylor College of Medicine, Houston, Texas

National Cancer Institute, Bethesda, Md.

National Institutes of Health, Bethesda, Md.

Queen Mary, University of London, London, U.K.

Dresden University of Technology, Dresden, Germany

University of Louisville, Louisville, Ky.

University of Louisville Research Foundation, Louisville, Ky.

University of Louisville School of Medicine, Louisville, Ky.

University of Pennsylvania, Philadelphia, Pa.

University of Toronto, Toronto, Ontario, Canada

University of Ulsan, Seoul, South Korea 